\title{
Dynamic Redeployment Coverage Location Model with Two Types of Servers
}

\author{
Huda Zaki Naji \& Noraida Abdul Ghani
}

\begin{abstract}
Designing the emergency medical services systems (EMS) are important in human societies, principally in urban areas where incidents occur at a high rate due to high population density. As such, the EMS managers face the task of searching for ways to improve the performance of the systems to respond to the requirements of patients taking into consideration the budget imposed by the state. This paper proposes an extension of the Dynamic Redeployment Coverage Location (DRCL) model to locate ambulances that are offering two types of services, i.e., Advance Life Support (ALS) and Basic Life Support (BLS) for two types of incoming calls (critical and non-critical). Apart from providing coverage for the non-critical calls, the BLS acts as a "backup" for the ALS in providing coverage to critical calls. The objective is to minimize the number of ambulances while meeting a predetermined level of coverage required.
\end{abstract}

Keywords-coverage location problem, emergency medical system EMS, hypercube model, queuing theory

\section{Introduction}

The main target of emergency medical service systems (EMS) is to reduce mortality rate, disability, and suffering in persons $[1,2]$. As such, EMS providers and managers are often faced with the difficult mission of locating a limited number of ambulances in a manner that will result in the best service to a constituent population [3, 4]. One of the main challenges for the EMS providers and managers is to adequately increase service coverage [5]. The dynamic redeployment of the ambulances is one strategy that will likely help increase coverage when some of the ambulances are busy. The EMS often receives many types of demand for the service and it provides multiple levels of care in emergency situations. Emergency medical calls are typically classified into two categories: critical and non-critical. The critical consists of calls that are likely to be "lifethreatening", while the non-critical are calls which are considered emergent but "non-life threatening".

Huda Zaki Naji

Department of Mathematics, College of Science, University of Basrah Iraq

School of Distance Education, University Sains Malaysia, 1800 Penang Malaysia

Noraida Abdul Ghani

School of Distance Education, University Sains Malaysia, 1800 Penang Malaysia
The EMS ambulance services can also be classified into two categories: advanced life support (ALS) and basic life support (BLS). ALS ambulances are managed by paramedics and are equipped to effectively handle critical demands such as cardiac resuscitation and airway management. BLS ambulances are managed by emergency medical technician (EMT) and are equipped for non-critical problems.

This paper proposes an extension of the Dynamic Redeployment Coverage Location (DRCL) model, to locate ambulances that are offering two types of services, i.e., Advance Life Support (ALS) and Basic Life Support (BLS) for two types of incoming calls (critical and non-critical). Apart from providing coverage for the non-critical calls, the BLS acts as a "backup" for the ALS in providing coverage to critical calls. The objective is to minimize the number of ambulances while meeting a predetermined level of coverage required.

This paper is organized as the follows. A literature review of the location models used in the EMS is discussed in section II. Section III presents the mathematical program of the model in which the two types of server (ALS \& BLS) and the two types of demand calls (critical \& non-critical) are incorporated. Conclusions and suggestions for future work are discussed in section IV.

\section{Literature review}

During the last two decades, many types of emergency location system models have evolved, in an attempt to formulate the emergency response systems in the best possible way. The emergency location system models are generally grouped into two broad categories: deterministic static and probabilistic static. The first model in deterministic static models is the Set Covering Location Problem (SCLP) introduced by Toregas, Swain, ReVelle and Bergman [6]. Its objective is to minimize the total cost of the EMS ambulances with the given distance or time threshold. SCLP ignores many aspects of the real problems. It makes no difference between the nodes on the basis of the volume of demand. So that, each node must be covered without regard to the cost. A few years later, Church and ReVelle [7] proposed the Maximum Coverage Location Problem (MCLP) model to counter some of the shortcomings of the SCLP. Its objective is to maximize the demand coverage given a limited number of the EMS ambulances.

All deterministic static models do not take into account the possibility that the server is busy when a call comes in; this is the main drawback for these models. Therefore, researchers used probabilistic static models as they admit the possibility that the server may be busy when a call comes in. 
The earlier models in probabilistic static models are the Maximum Expected Coverage Location Problem (MEXCLP) introduced by Daskin [8] and the Maximum Availability Location Problem (MALP) introduced by ReVelle and Hogan [9, 10]. Its objective maximizes the expected and available coverage, respectively. A common assumption used in these models is the server independence and system-wide server busy probability. There are two versions of MALP: MALP I and MALP II. The original model MALP I supposed that the EMS ambulances had the same busy probability fraction while the MALP II supposed a local busy probability fraction that is associated to the demand node. Galvão, Chiyoshi and Morabito [11] gave a unified view of Daskin's MEXCLP and ReVelle and Hogan's MALP I. They developed an extension of MALP I by dropping the simplifying assumptions of the original model (independence among servers and system-wide server busy probability). This is done by embedding the Larson's hypercube model [12].

To relax the assumption of the independence of busy probability of the various servers in the MALP I, Marianov and ReVelle [13] developed the Queuing Maximum Availability Location Problem (Q-MALP). To derive the busy probability of the servers at each demand node, the QMALP modeled the arrivals and services at each node as an M/G/s/s queuing system. Later, Noraida [14] extended the Q-MALP model by formulating the Multi-server Queuing Maximum Availability Location Problem (MQ-MALP). Its objective maximizes the number of demand nodes that are covered by two types of services, i.e., the Advance Life Support (ALS) and Basic Life Support (BLS) with reliability level. In addition, this model takes into account the stochastic nature of the travel times.

All previously mentioned models (Deterministic and Probabilistic Static) are useful in the strategic level but lack the flexibility in the operational level because they do not take relocation into account. Demand patterns in many areas are not static but changing during different times of the day. Thus, the decision makers need to relocate some facilities in order not to leave any areas without coverage. The two previous papers on relocation in the EMS literature are by Repede and Bernardo [15] and by Gendreau, Laporte and Semet [16]. Repede, et al. [15], formulated the Time Maximum Expected Coverage Location Problem (TIMEXCLP) model, which is an extension of MEXCLP. Its objective is to maximize the expected coverage for different time intervals. Gendreau, et al. [16], on the other hand, formulated the Dynamic Double Standard Model (DDSMt) that looked at actual demand changes.

Later, Rajagopalan [17] formulated three alternative models which are: the Dynamic Set Covering Problem (DSCP) to minimize the number of EMS ambulances at different time intervals, the Minimum Redeployment Location Problem (MRLP) to minimize the number of EMS vehicles and their redeployments and the Minimum Service Time with Constant Coverage (MSTCC) to minimize the response time required to reach each call. Rajagopalan, Saydam \& Xiao [18] formulated the Dynamic Available Coverage Location (DACL) model with the objective of determining the minimum number of the EMS vehicles and their locations. Recently, Saydam, et al. [5] formulated the Dynamic Redeployment Coverage Location model (DRCL), which is an extension of the DACL model by simultaneously minimizing both the number of EMS vehicle and redeployments. This model assumed there is only one type of vehicle and the nearest available vehicle dispatch policy.

In this paper we propose an extension of the DRCL model to locate ambulances offering two types of services, i.e., the ALS and BLS that cater to two different types of incoming calls (critical and non-critical), hence forth known as Multi-server DRCL (MS-DRCL). The objective is to minimize the number of ambulances while meeting a predetermined level of coverage required.

\section{The MS-DRCL Model}

In this section MS-DRCL model is formulated to determine the minimum number of ambulances with two types of medical units and catering to two types of calls in a geographical area. The following list summarizes the symbols used.

$i=$ the number of nodes in the system, $i=1,2, \cdots, n$

$j=$ the number of medical units site in the system, $j=1,2, \cdots, m$

$t=$ the index of time intervals $(t=1,2, \cdots, T)$

$J_{t}=$ the set of node which contains medical units at time $t$

$m_{t}^{A}=$ the total number of ALS units at time $t$

$m_{t}^{B}=$ the total number of BLS units at time $t$

$\alpha_{t}=$ the available coverage at time $t$

$i=$ the number of nodes in the system, $i=1, \cdots, n$

$j=$ the number of ambulances site in the system, $j=1, \cdots, m$

$t=$ the index of time intervals, $t=1, \cdots, T$

$m_{t}^{A}=$ the total number of ALS ambulances at time $t$

$m_{t}^{B}=$ the total number of BLS ambulances at time $t$

$\alpha_{t}=$ the available coverage at time $t$

$h_{i, t}^{A}, h_{i, t}^{B}=$ the fraction of totalcritical and non - critical demand at node $i$ at time $t$, respectively.

$c_{t}=$ the minimum expected coverage requiremen $\mathrm{t}$ at time $t$

$\rho_{i, t}^{A}, \rho_{i, t}^{B}=$ the busy probability of ALS and BLS ambulances at node $i$ at time $t$, respectively.

$\rho_{t}^{A}, \rho_{t}^{B}=$ the average busy probability of ALS and BLS ambulances at time $t$, respectively.

$P_{0}^{A}, P_{0}^{B}=$ the probability that all ambulances of typeALS and BLS are free in $M / M / m /$ loss sy stem,respectively.

$P^{A}, P^{B}=$ the probability that all ambulances of typeALS and BLS are busy in $M / M / m /$ loss system,respectively.

$Q^{A}\left(m_{t}^{A}, \rho_{t}^{A}, j\right)=$ the correction factor for Jarvis' algorithm [20] adjusts that probability for ALS ambulances cooperation

$Q^{B}\left(m_{t}^{B}, \rho_{t}^{B}, j\right)=$ the correction factor for Jarvis' algorithm [20] adjusts that probability for BLS ambulances cooperation 
Essentially, the approximation for the hypercube model given by Larson [12, 19] and its extension by Jarvis [20] assumes servers operate independently but then correct this obvious "error" with the correction factor. Hence, the correction factor of ALS ambulances, $Q^{A}\left(m_{t}^{A}, \rho_{t}^{A}, j\right)$ and correction factor of BLS ambulances, $Q^{B}\left(m_{t}^{B}, \rho_{t}^{B}, j\right)$ are given by the following equations:

$Q^{A}\left(m_{t}^{A}, \rho_{t}^{A}, j\right)=\frac{\sum_{k=j}^{m_{t}^{A}-1}\left(m_{t}^{A}-j-1\right) !\left(m_{t}^{A}-k\right)\left(\rho_{t}^{A^{k-j}}\right) P_{0}^{A}}{(k-j) !\left(1-P^{A}\right)^{j}\left(m_{t}^{A}\right) !}$
$, \quad \forall j=0,1, \cdots, m_{t}^{A}-1$

$Q^{B}\left(m_{t}^{B}, \rho_{t}^{B}, j\right)=\frac{\sum_{k=j}^{m_{t}^{B}-1}\left(m_{t}^{B}-j-1\right) !\left(m_{t}^{B}-k\right)\left(\rho_{t}^{B^{k-j}}\right) P_{0}^{B}}{(k-j) !\left(1-P^{B}\right)^{j}\left(m_{t}^{B}\right) !}$

, $\forall j=0,1, \cdots, m_{t}^{B}-1$

Also we define

$x_{i, j, t}^{A}=\left\{\begin{array}{l}1, \text { if the ALS ambulance } j \text { is in node } i \text { at time } t \\ 0, \text { otherwise }\end{array}\right.$

$x_{i, j, t}^{B}=\left\{\begin{array}{l}1, \text { if the BLS ambulance } j \text { is in node } i \text { at time } t \\ 0, \text { otherwise }\end{array}\right.$

$y_{i, t}^{A}=\left\{\begin{array}{l}1, \text { if at least one ALS or BLS ambulance cover node } \\ \quad \text { with } \alpha_{t} \text { reliabilit } \mathrm{y} \text { at time } t \\ 0, \text { otherwise }\end{array}\right.$

$y_{i, t}^{B}=\left\{\begin{array}{l}1, \text { if at least one BLS ambulance cover node } i \\ \quad \text { with } \alpha_{t} \text { reliabilit y at time } t \\ 0, \text { otherwise }\end{array}\right.$

With these definitions, the objective of minimizing the number of ALS ambulances while meeting a predetermined level of coverage requirement can be formulated as

$$
\sum_{t=1}^{T} \sum_{i=1}^{n} \sum_{j \in J_{t}} x_{i, j, t}^{A}
$$

and the objective of minimizing the number of BLS ambulances while meeting a predetermined level of coverage requirement can be formulated as

$$
\sum_{t=1}^{T} \sum_{i=1}^{n} \sum_{j \in J_{t}} x_{i, j, t}^{B}
$$

Thus, the objective function becomes

$$
\text { Min } Z=\sum_{t=1}^{T} \sum_{i=1}^{n} \sum_{j \in J_{t}} x_{i, j, t}^{A}+\sum_{t=1}^{T} \sum_{i=1}^{n} \sum_{j \in J_{t}} x_{i, j, t}^{B}
$$

Thus, the MR-DRCL can be formulated as

$$
\operatorname{Min} \quad Z=\sum_{t=1}^{T} \sum_{i=1}^{n} \sum_{j \in J_{t}} x_{i, j, t}^{A}+\sum_{t=1}^{T} \sum_{i=1}^{n} \sum_{j \in J_{t}} x_{i, j, t}^{B}
$$

Subject to:

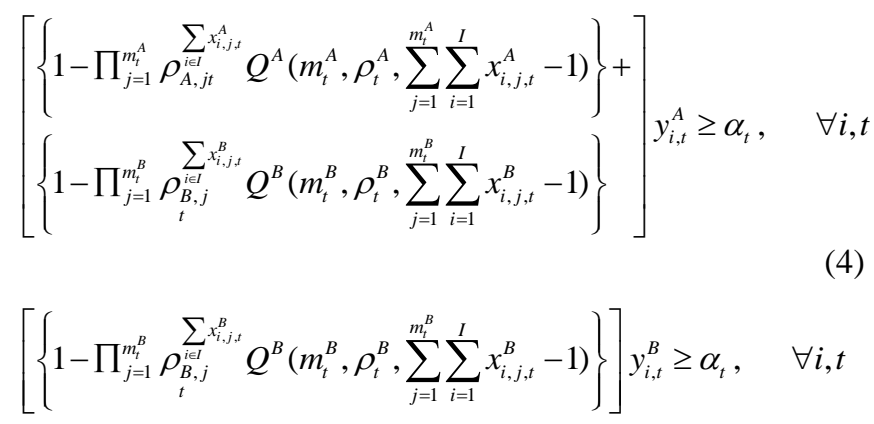

$\sum_{i \in I} h_{i, t}^{A} y_{i, t}^{A}+\sum_{i \in I} h_{i, t}^{B} y_{i, t}^{B} \geq c_{t}, \quad \forall t$

$\sum_{i=1}^{n} \sum_{j \in J_{t}} x_{i, j, t}^{A} \leq m_{t}^{A}, \quad \forall t$

$\sum_{i=1}^{n} \sum_{j \in J_{t}} x_{i, j, t}^{B} \leq m_{t}^{B}, \quad \forall t$

$x_{i, j, t}^{A}, x_{i, j, t}^{B}, y_{i, t}^{A}, y_{i, t}^{B}=\{0,1\}, \quad \forall i, j, t$

The objective (3) minimizes the total number of ambulances (ALS and BLS) while meeting a predetermined level of coverage requirement. The constraint (4) ensures the nodes which are covered by ALS or BLS ambulances are within the required $\alpha_{t}$ reliability. The constraint (5) ensures the nodes which are covered by BLS are within the required $\alpha_{t}$ reliability. The constraint (6) ensures the total coverage of critical calls that are covered by the ALS or BLS ambulances and non-critical calls that are covered by the BLS units cannot be less than the minimum percentage coverage at time $t,\left(c_{t}\right)$. The constraints (7) and (8) limit the number of ALS and BLS ambulances at time interval $t$, respectively. Finally, the constraint (9) forces the variables to be binary (i.e. 0,1$)$.

\section{CONCLUSIONS}

In this paper, we highlight the most important literature related to our work that has been used during the past two decades in the EMS, together with the advantages and limitations of their work. To fill the gap in the literature, we propose the MS-DRCL to minimize the number of ambulances while meeting a predetermined level of coverage requirement. Our model extends the DRCL model with two types of servers, namely the ALS and BLS ambulances, which are available for responding to two types of emergency medical calls, namely the critical and noncritical calls. Future work will be to solve our model via the meta-heuristic methods. 


\section{References}

[1] L. L. Brotcorne, and G. Laporte, and F. Semet, "Ambulance location and relocation models," European Journal of Operational Research, vol. 147, pp. 451-63, 2003.

[2] J. Goldberg, "Operations research models for the deployment of emergency services vehicles," EMS Management Journal, 1, vol. 1, pp. 20-39, 2004.

[3] H. K. Rajagopalan, and C. Saydam, and J. Xiao, "A multiperiod expected covering location model for dynamic redeployment of ambulances," in conference-10th EWGT meeting and 16th MiniEURO, Poznan, Poland, 2005.

[4] H. K. Rajagopalan, and F. E. Vergara, and C. Saydam, and J. Xiao, "Developing effective meta-heuristics for a probabilistic location model via experimental design," European Journal of Operation Research, vol. 177, pp. 83-101, 2007.

[5] C. Saydam , and H. K. Rajagopalan, and E. Sharer, and K. L. Belanger, "The dynamic redeployment coverage location model," Operational Research Society Ltd, vol. 2, pp. 103-119, 2013.

[6] C. Toregas, and R. Swain, and C. ReVelle, and L. Bergman, "The location of emergency service facilities," Operation Research, vol. 19, pp. 1363-1373, 1971

[7] R. L. Church, and C. S. ReVelle, "The maximum covering location problem," Papers of Regional Science Association, vol. 32, pp. 101118, 1974.

[8] M. S. Daskin, "A maximum expected covering location model: Formulation, properties and heuristic solution," Trans Science, vol. 17, pp. 48-68, 1983.

[9] C. ReVelle, and K. Hogan, "The maximum availability location problem," Trans Science, vol. 23, pp. 192-200, 1989.

[10] C. ReVelle, and K. Hogan, "The maximum reliability location problem and alpha reliable p-center problems: derivatives of the probabilistic location set covering problem," Annals of Operation Research, vol. 18, pp. 155-174, 1989.

[11] R. D. Galvao, and F. Y. Chiyoshi, and R. Morabito, "Towards unified formulations and extensions of two classical probabilistic location models," Computers and Operations Research, in press.

[12] R. C. Larson, "A hypercube queuing model for facility location and redistricting in urban emergency services," Computers and Operations Research, vol. 1, pp. 67-95, 1974.

[13] V. Marianov, and C. ReVelle, "The queueing maximal availability location problem: a model for the siting of emergency vehicles," European Journal of Operation Research, 93, vol. 1, pp. 110-120, 1996.

[14] A. G. Noraida, "Multi-server Queuing Maximum Availability Location Problem with Stochastic Travel Times," in Proceedings of the World Congress on Engineering, London, U. K vol. I, 2012.

[15] J. F. Repede, and J. J. Bernardo, "Developing and validating a decision support system for locating emergency medical vehicles in louisville kentucky," European Journal of Operation Research, 75, vol. 5, pp. 567-581, 1994.

[16] M. Gendreau, and G. Laporte, and F. Semet, "A dynamic model and parallel tabu search heuristic for real-time ambulance relocation," Parallel Computers, vol. 27, pp. 1641-1653, 2001

[17] Rajagopalan HK, Vergara FE, Saydam C, Xiao J. "Developing and validating realistic dynamic relocation models for emergency medical systems : a hybrid meta-heuristic approach," Ph.D. dissertation, Business Information System and Operations Management Department, University of north Carolina, Charlotte, 2006.

[18] H. K. Rajagopalan, and C. Saydam, and J. Xiao, "A multiperiod set covering location model for dynamic redeployment of ambulances," Computers and Operations Research, 35, vol. 3, pp. 814-826, 2008.

[19] R. C. Larson, "Approximating the performance of urban emergency service systems," Operations Research, vol. 23, pp. 845-68, 1975.

[20] J. P. Jarvis, "Approximating the equilibrium behavior of multi-server loss systems," Management Science, vol. 31, pp. 235-239, 1985. 